\title{
Hepatite B na Amazônia: revisão integrativa
}

\author{
Hepatites B in Amazon: integrative review
}

Hepatitis B en la Amazonía: revisión integrativa

\begin{abstract}
Raissa de Sousa Marinho Pimenta ${ }^{1 *}$, Natália Pezzin Guinhazi ${ }^{1}$, Raphaela Antunes Coelho ${ }^{1}$, Alice Frazão Costa ${ }^{1}$, Ana Carolina Furtado Ribeiro Baetas ${ }^{1}$, Andressa Viana Oliveira ${ }^{1}$, Ana Paula Santos Oliveira Brito ${ }^{1}$, Herbert Paulino Cordeiro ${ }^{1}$ e Hamilton Cezar Rocha Garcia ${ }^{1}$.
\end{abstract}

\section{RESUMO}

Objetivo: Realizar uma revisão integrativa de artigos voltados para os casos de hepatite B na Amazônia. Métodos: Este estudo consiste em uma revisão da literatura, realizada entre 2013 e 2019, na qual realizouse consulta a artigos científicos em língua inglesa, portuguesa e espanhola, selecionados através de busca no banco de dados do Scielo e da Bireme, a partir das fontes Medline e Lilacs. Resultados: Foram selecionados 26 artigos e todos atrelam às altas taxas de prevalência da infecção pelo vírus da hepatite $B$ na Amazônia. Dentre esses, 5 artigos abordam sobre a prevalência da infecção nos estados do Pará, Acre, Rondônia e Amazonas, 7 artigos focam em aspectos epidemiológicos e clínicos, 4 se pautam no diagnóstico, 5 sobre os diferentes tratamentos e 5 abordam sobre medidas profiláticas. Considerações finais: Concluise que a Hepatite B é um grave problema de saúde no Brasil, sobretudo na região amazônica, por conta de imunização ausente ou incompleta, embora as vacinas protetoras contra a hepatite B tenham sido introduzidas. Por isso, há necessidade de reavaliação dos programas já instituídos e implementação de novas políticas a fim de reduzir a hepatite $B$ na população.

Palavras-chaves: Hepatite B, Epidemiologia, Ecossistema amazônico.

\begin{abstract}
Objective: To carry out a integrative review of articles focusing on cases of hepatites $B$ in Amazon. Methods: This study constitutes a review of literature, conducted between 2013 and 2019, were consulted scientific articles in English, Portuguese and Spanish, selected through the search of the scielo and bireme database, from the sources Medline and Lilacs. Results: 26 articles were selected and all of them link about the high rates of prevalence of hepatitis $B$ virus infection in the Amazon. Among these, 5 articles deal with the prevalence of infection in the states of Pará, Acre, Rondônia and Amazonas, 7 articles focus on epidemiological and clinical aspects, 4 in diagnosis, 5 on the different treatments and 5 on prophylactic measures. Final considerations: It was concluded that hepatitis B is a serious health problem in Brazil, especially in the Amazon region, due to missing or incomplete immunization, although the protective vaccines against hepatitis B have been introduced. Therefore, there is a need for reassessment of programs already instituted and implementation of new policies in order to reduce hepatitis B in the population.
\end{abstract}

Keywords: Hepatitis B, Epidemiology, Amazonian ecosystem.

1 Centro Universitário Metropolitano da Amazônia (UNIFAMAZ), Belém - PA.

*E-mail: raissasmpimenta@gmail.com 


\section{RESUMEN}

Objetivo: Realizar una revisión integrativa de artículos centrados en casos de hepatitis B en la Amazonía. Métodos: Este estudio consiste en una revisión de la literatura, realizadas entre 2013 y 2019, en la que consultamos artículos científicos en inglés, portugués y español, seleccionados mediante lá búsqueda en las bases de datos Scielo y Bireme de las fuentes Medline y Lilacs. Resultados: Seleccionaron 26 artículos y todos se relacionan com las tasas de prevalencia de la infección por el virus de la hepatitis B en la Amazonía. Entre estos, 5 artículos abordan la prevalencia de la infección en los estados de Pará, Acre, Rondônia y Amazonas, 7 artículos se centran en aspectos epidemiológicos y clínicos, 4 se basan en el diagnóstico, 5 en diferentes tratamientos y 5 se refieren a medidas profilácticas. Consideraciones finales: Concluye que la hepatitis $B$ es un problema de salud grave en Brasil, especialmente en la región amazónica, debido a la inmunización incompleta o faltante, aunque se han introducido vacunas protectoras contra la hepatitis B. Por lo tanto, es necesario reevaluar los programas existentes e implementar nuevas políticas para reducir la hepatitis $B$ en la población.

Palabras clave: Hepatitis B, Epidemiología, Ecosistema amazónico.

\section{INTRODUÇÃO}

A hepatite $B$ foi reconhecida há, aproximadamente, 130 anos, através de registros realizados durante uma investigação de casos de icterícia pós-vacinação antivariólica em trabalhadores do estaleiro de Bremen, na Alemanha, despertando o interesse de pesquisadores desde então (NUNES HM, 2017).

O vírus da hepatite B (HBV) pertence à família Hepadnaviridae, gênero Orthohepadnavirus, espécie Hepatitis B vírus (NUNES HM, 2017). Vale ressaltar que apenas este gênero citado engloba os vírus que infectam mamíferos, embora o HBV seja capaz de infectar humanos e outros primatas superiores não humanos, o que é menos frequente (BRASIL, 2017).

É considerada uma doença infecciosa viral transmitida diretamente de indivíduo a indivíduo através de soluções de continuidade (contato com sangue, exsudatos e outros fluidos corporais). As vias naturais de transmissão são as principais responsáveis pela alta prevalência da doença, incluindo transmissão sexual, vertical (perinatal e intrauterina) e horizontal por meio de exposição ambiental em casa, nas prisões e outras instituições de confinamento. No caso da transmissão vertical, vale ressaltar que a gestante portadora de um perfil sorológico HBsAg/HBeAg positivo, aumenta o risco de infecção do recém-nascido (BRASIL, 2019).

Dessa forma, a hepatite B é um grave problema de saúde pública, permanecendo com altos índices de morbidade e mortalidade por sua alta infectividade e prevalência em populações tradicionais com menor complexidade urbana, como na Região Amazônica, podendo ser estabelecida uma relação com as diferentes realidades socioeconômicas do território brasileiro (BRASIL, 2017; SOUZA FO, 2019). A Organização Mundial da Saúde (OMS) estima que cerca de 2 bilhões de pessoas já se infectaram pelo vírus da hepatite $B$, e destes, 350 milhões são portadores crônicos, os quais apresentam alto risco de óbito por cirrose hepática e câncer de fígado (SILVA RSU, 2017).

O HBV é considerado um vírus oncogênico, capaz de ocasionar ou contribuir para o surgimento de tumores, e apresenta dez genótipos, classificados de A a J, que diferem um do outro em sequências do genoma viral em mais de $8 \%$. Esses genótipos são diferentes entre si pela sequência de nucleotídeos no genoma, patogenicidade e distribuição geográfica, além de que podem ser divididos em subgenótipos. De acordo com o panorama mundial, o genótipo $A$ apresenta risco maior para cronicidade e é mais frequente na África Subsaariana, no Norte da Europa e na África Ocidental; os genótipos B e C são característicos da Ásia; o D predomina na África, Europa, países do Mediterrâneo e Índia; o E na África Central e na Arábia Saudita; o F na Tunísia, Brasil e Espanha; o G na França, Alemanha e EUA; o H na América do Sul e América Central; o I no Vietnã e Laos; e por fim, o J, encontrado nas ilhas do Japão (NUNES HM, 2017). Em relação ao Brasil, especificamente na região amazônica brasileira, o genótipo $A$ é o mais comum, seguido pelos genótipos $D$ e F, sendo este último mais prevalente nas comunidades indígenas (SANTANA CC, 2018). 
A hepatite B é considerada a segunda maior causa de óbitos entre as hepatites virais. Entre os anos de 2000 até 2017, foram identificados 15.033 óbitos; desses, 54,8\% tiveram a hepatite B como causa base, sendo a maior parte na região Sudeste $(41,5 \%)$. No entanto, a região Norte foi a que apresentou os maiores índices de mortalidade em todo o período, chegando a 0,4 óbitos por 100 mil habitantes no ano de 2017 (BRASIL, 2019).

Diante de um novo boletim epidemiológico publicado, o cenário não sofreu alterações. Durante o período de 1999-2018, foram notificados no Sistema de Informações de Agravos de Notificação (Sinan) 623.814 casos confirmados de hepatites virais no Brasil, sendo que destes, $233.027(36,8 \%)$ eram referentes à hepatite $B$, permanecendo esta, ainda, com a maior prevalência no país (BRASIL, 2019).

A endemicidade da hepatite B pode ser avaliada pela prevalência de portadores do HBsAg, ou com evidência sorológica de infecção prévia, com a classificação da endemicidade mundial em 3 padrões: baixa $(<1 \%)$, intermediária (1 a $5 \%)$ e alta $(>5 \%)$. A soropositividade para o HBsAg varia entre regiões e grupos populacionais específicos (SOUTO FJD, 2016).

No final do século XX, o Brasil foi classificado como tendo moderada endemicidade em relação à hepatite B. Há muito se sabia que a prevalência da hepatite $B$ aumentava do sul para o norte do país. A região amazônica tem a maior endemicidade no Brasil, particularmente no Acre, no sul da Amazônia, assim como em Rondônia, Pará e Noroeste do Mato Grosso (SOUTO FJD, 2016). De modo histórico, o Brasil, apresenta diversas diferenças em seus dados epidemiológicos, isso porque há diferentes medidas de acompanhamento e controle de danos e agravos infecciosos (SOUZA FO, 2019).

Diante do cenário mundial, a infecção pelo HBV continua sendo um problema de saúde pública devido à alta transmissibilidade, embora sua epidemiologia venha se modificando ao longo do tempo, devido vacinação em larga escala e políticas de testagem para a doença em bancos de sangue e na população em geral (BRASIL, 2019).

No Brasil, apesar da introdução da vacina na Amazônia, em 1989, e dos esforços em imunização e prevenção no Sistema Único de Saúde (SUS), com a produção nacional autossuficiente de vacinas, a transmissão da hepatite B ainda é uma realidade. Cerca de 17.000 novos casos são detectados e notificados anualmente, o que contribui para evidenciar o impacto da doença no território brasileiro (BRASIL, 2017).

À vista disso, este trabalho justifica-se pelo fato de que apesar do progresso e da existência de várias medidas profiláticas instituídas, visando diminuir a incidência da hepatite B na Amazônia, ainda há grande desconhecimento sobre o impacto da infecção na população. Deste modo, este trabalho objetiva realizar uma revisão integrativa de artigos voltados para a prevalência e incidência de hepatite $B$ na região amazônica.

\section{MÉTODOS}

Foi efetuada uma revisão integrativa da literatura, com intuito de capturar artigos que apresentassem em seus desenhos de estudo correlação com o tema, Hepatite B na Amazônia. Foram selecionados estudos publicados de 2013 a 2019 nas línguas inglesa, portuguesa e espanhola.

Os descritores utilizados foram Hepatite B, Epidemiologia e Amazônia. As bases de dados consultadas foram Scientific Electronic Library Online (Scielo), Centro Latino-Americano e do Caribe de Informação em Ciências da Saúde (Bireme), Sistema Online de Busca e Análise de Literatura Médica (Medline), Literatura Latino-Americana e do Caribe em Ciências da Saúde (Lilacs), Pubmed e a biblioteca do Cochrane. A pesquisa dos artigos foi realizada entre novembro e dezembro de 2019.

Foram encontrados 45 artigos, porém, apenas 26 destes, se encontravam dentro dos critérios de elegibilidade. Os critérios de inclusão para os estudos encontrados foram à abordagem dos casos de hepatite B na Amazônia, e estudos comparativos entre os mesmos. Como critérios de exclusão, adotou-se não analisar artigos com informações incompletas ou que não abordaram a região Amazônica e estudos fora do intervalo determinado e sem relevância para a pesquisa (Figura 1). 
Figura 1 - Fluxograma demonstrando critérios de exclusão e inclusão para a pesquisa.

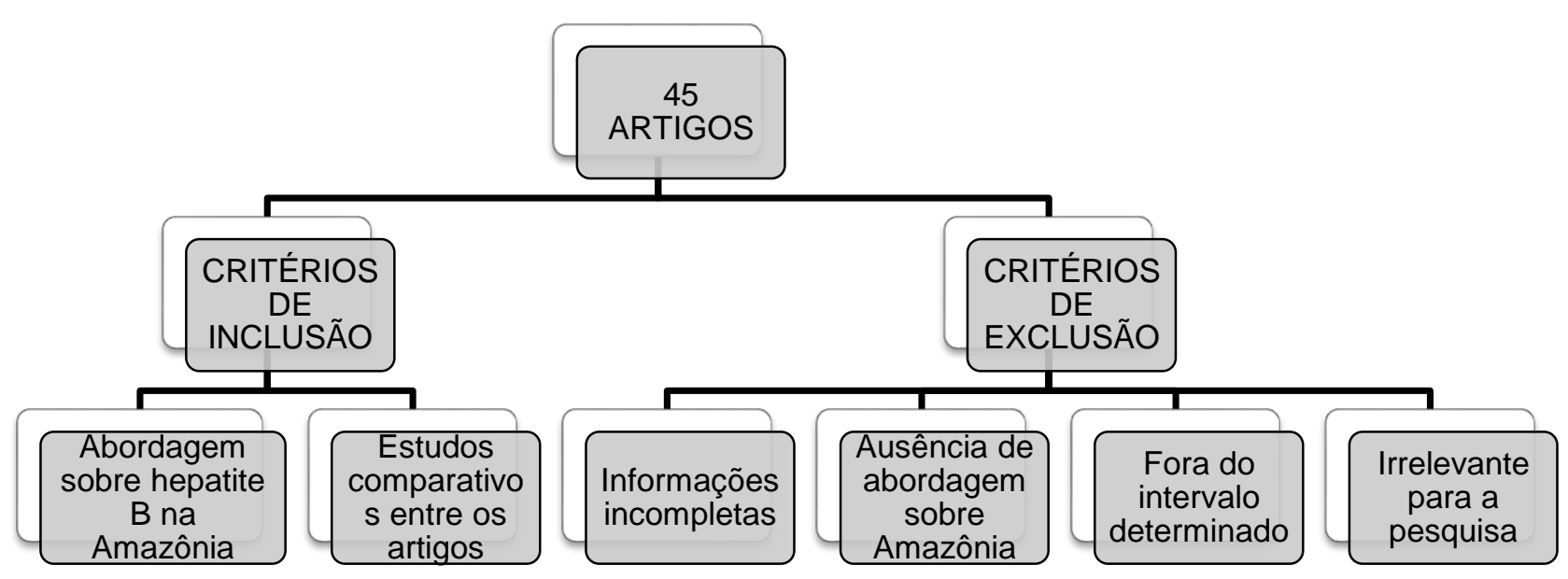

Fonte: Pimenta RSM, et al., 2020

\section{RESULTADOS}

Depois de aplicados os critérios de elegibilidade, 26 artigos foram identificados no período do estudo, publicados a partir de 2013, sendo sete destes, publicados a partir de 2018, demonstrando que o tema ainda é atual. Quanto ao local de procedência dos artigos, em sua maioria foram publicados na América do Sul.

Dentre os 26 artigos selecionados, a maioria se refere às altas taxas de incidência e prevalência da infecção pelo vírus da hepatite B na região Norte, em especial a Amazônia, esclarecendo a relação entre as condições sanitárias e a prevalência da infecção. Destes, cinco artigos abordam sobre a prevalência da infecção na região amazônica, incluindo, principalmente os estados do Pará, Acre, Rondônia e Amazonas.

Além disso, sete focam em aspectos epidemiológicos e clínicos observados em pacientes acometidos não só na região Norte, como também no Brasil em geral. Somando-se aos outros artigos utilizados, quatro se pautam no diagnóstico mais aprofundado, abordando a caracterização molecular do vírus na população da Amazônia ocidental brasileira. E por fim, cinco abordam sobre os diferentes tratamentos existentes, enquanto que cinco artigos abordam sobre medidas profiláticas, como o desenvolvimento de vacina contra a hepatite $\mathrm{B}$, relacionando-a, paradoxalmente, com os altos índices de infecção na região amazônica, mesmo após a implantação (Quadro 1). 
Quadro 1 - Caracterização dos estudos científicos (observacionais e experimentais) incluídos na revisão.

\begin{tabular}{|c|c|c|c|c|c|}
\hline Título & Local & Autor/ Ano & Revista & Desenho & Desfecho/Resultados \\
\hline $\begin{array}{l}\text { Levantamento de dados entre o } \\
\text { período de } 2010 \text { a } 2016 \text { de hepatite B } \\
\text { no município de Rolim de Moura. }\end{array}$ & $\begin{array}{l}\text { Rondônia (Rolim de } \\
\text { Moura). }\end{array}$ & $\begin{array}{l}\text { CRUZ AS, et al., } \\
2018 .\end{array}$ & Braz J Surg Clin Res. & $\begin{array}{l}\text { Transversal } \\
\text { (incidência). }\end{array}$ & $\begin{array}{l}\text { Incidência média anual de } \\
\text { hepatite } B: 2,75 / 10.000 \text { hab. }\end{array}$ \\
\hline $\begin{array}{l}\text { As hepatites virais no brasil: uma } \\
\text { análise a partir dos seus territórios. }\end{array}$ & Nacional & $\begin{array}{l}\text { FARIAS CS, et al., } \\
2019 .\end{array}$ & Raega. & $\begin{array}{l}\text { Transversal } \\
\text { (incidência). }\end{array}$ & $\begin{array}{l}\text { Maiores Incidências médias } \\
\text { anuais de hepatite B: } \\
\text { Acre - 7,94/10.000 hab. } \\
\text { Rondônia - 2,62/10.000 hab. }\end{array}$ \\
\hline $\begin{array}{l}\text { Especificidades e desafios das } \\
\text { políticas públicas de saúde na } \\
\text { Amazônia. }\end{array}$ & Manaus & GARNELO L, 2019. & Cad. Saúde Público. & Ecológico. & $\begin{array}{l}\text { Desigualdades entre as } \\
\text { populações residentes na } \\
\text { Amazônia e principais } \\
\text { dificuldades enfrentadas. }\end{array}$ \\
\hline $\begin{array}{c}\text { Análise do perfil sociodemográfico de } \\
\text { notificados para hepatite B e } \\
\text { imunização contra a doença. }\end{array}$ & $\begin{array}{l}\text { Minas Gerais (Montes } \\
\text { Claros). }\end{array}$ & $\begin{array}{l}\text { GUSMÃO BM, et al., } \\
2017 .\end{array}$ & J res: fundam care. & $\begin{array}{l}\text { Transversal } \\
\text { (prevalência). }\end{array}$ & $\begin{array}{c}\text { Prevalência de hepatite B } \\
\text { 3,39/10.000 hab., período 2007- } \\
\text { 2015. }\end{array}$ \\
\hline $\begin{array}{l}\text { Treatment of chronic hepatitis B naïve } \\
\text { patients with a therapeutic vaccine } \\
\text { containing HBs and HBc antigens (a } \\
\text { randomized, open and treatment } \\
\text { controlled phase III clinical trial). }\end{array}$ & Bangladesh. & $\begin{array}{l}\text { MAHTAB MAI, et al., } \\
2018 .\end{array}$ & PLOS ONE. & $\begin{array}{l}\text { Ensaio } \\
\text { clínico fase } \\
\text { III. }\end{array}$ & $\begin{array}{c}\text { Controle da infecção crônica por } \\
\text { HBV mais prolongado na nova } \\
\text { vacina proposta, que na vacina } \\
\text { padrão, e houve menos eventos } \\
\text { adversos. }\end{array}$ \\
\hline $\begin{array}{l}\text { Fatores associados à imunização } \\
\text { contra Hepatite B entre trabalhadores } \\
\text { da Estratégia Saúde da Família. }\end{array}$ & $\begin{array}{c}\text { Minas Gerais (Montes } \\
\text { Claros). }\end{array}$ & $\begin{array}{l}\text { MARTINS AMEBL, } \\
\quad \text { et al., } 2015 .\end{array}$ & Rev Bras Enferm. & $\begin{array}{l}\text { Transversal } \\
\text { (prevalência). }\end{array}$ & $\begin{array}{l}\text { Prevalência de } 52,5 \% \text { da } \\
\text { vacinação de três doses VHB, } \\
\text { entre } 761 \text { entrevistados. }\end{array}$ \\
\hline $\begin{array}{l}\text { Positive hepatitis B surface antigen } \\
\text { after vaccination in a newborn. }\end{array}$ & Portugal (Matosinhos). & $\begin{array}{l}\text { MARTINS M, et al., } \\
2018 .\end{array}$ & Nascer e Crescer. & $\begin{array}{l}\text { Relato de } \\
\text { caso. }\end{array}$ & $\begin{array}{c}\text { Descrição de um recém-nascido } \\
\text { prematuro falso positivo } \\
\text { transitório, de mãe vacinada. }\end{array}$ \\
\hline
\end{tabular}




\begin{tabular}{|c|c|c|c|c|c|}
\hline Título & Local & Autor/ Ano & Revista & Desenho & Desfecho/Resultados \\
\hline $\begin{array}{l}\text { Assessment of the prevalence of } \\
\text { vertical hepatitis } B \text { transmission in two } \\
\text { consecutive generations. }\end{array}$ & São Paulo (São Paulo). & $\begin{array}{l}\text { NAKANO LA, et al., } \\
2018 .\end{array}$ & Rev Assoc Med Bras. & $\begin{array}{l}\text { Transversal } \\
\text { (prevalência). }\end{array}$ & $\begin{array}{l}\text { Prevalência de transmissão de } \\
\text { VHB de mãe ao paciente de } \\
17,8 \% \text {, e do paciente para o } \\
\text { filho de } 7,9 \% \text {, entre } 101 \\
\text { pacientes. }\end{array}$ \\
\hline $\begin{array}{c}\text { Infecção oculta pelo vírus da hepatite } \\
\text { B em comunidade amazônica } \\
\text { submetida a intenso fluxo migratório, } \\
\text { estado do Pará, Brasil. }\end{array}$ & Pará (Juruti). & $\begin{array}{l}\text { NUNES HM, et al., } \\
2017 .\end{array}$ & Rev Pan-Amaz Saude. & $\begin{array}{l}\text { Transversal } \\
\text { (prevalência). }\end{array}$ & $\begin{array}{l}\text { Prevalência de infecção oculta } \\
\text { pelo VHB de } 0,1 \% \text { entre } 3991 \\
\text { indivíduos de um projeto sobre } \\
\text { hepatites virais. }\end{array}$ \\
\hline $\begin{array}{c}\text { Hepatitis B and Delta: clinical aspects } \\
\text { of patients in the Brazilian Western } \\
\text { Amazonia. }\end{array}$ & Acre (Cruzeiro do Sul). & $\begin{array}{l}\text { OLIVEIRA MS, et al., } \\
2019 .\end{array}$ & Rev Bras Enferm. & Transversal. & $\begin{array}{c}\text { Sinais e sintomas mais } \\
\text { frequentes na superinfecção } \\
\text { VHB+VHD que nos } \\
\text { monoinfectados (VHB). }\end{array}$ \\
\hline $\begin{array}{c}\text { Prevalence of occult hepatitis } B \text { in a } \\
\text { population from the Brazilian Amazon } \\
\text { region. }\end{array}$ & Pará (Belém). & $\begin{array}{l}\text { SANT'ANNA, et al., } \\
2018 .\end{array}$ & J Med Virol. & $\begin{array}{l}\text { Transversal } \\
\text { (prevalência). }\end{array}$ & $\begin{array}{c}\text { Prevalência de } 2,69 \% \text { de } \\
\text { infecção oculta pelo VHB entre } \\
965 \text { pacientes suspeitos de } \\
\text { infecção por VHB na triagem. }\end{array}$ \\
\hline $\begin{array}{c}\text { Prevalência da infecção pelo vírus da } \\
\text { hepatite B em um município do interior } \\
\text { do estado do Acre, Amazônia } \\
\text { Ocidental, Brasil. }\end{array}$ & Acre (Porto Acre). & $\begin{array}{l}\text { SILVA RSU, et al., } \\
2017 .\end{array}$ & Rev Pan-Amaz Saude. & $\begin{array}{l}\text { Transversal } \\
\text { (prevalência). }\end{array}$ & $\begin{array}{c}\text { Prevalência de VHB de } 2,17 \% \\
(217 / 10.000) \text { em pacientes de } \\
\text { demanda espontânea em um } \\
\text { posto de saúde. }\end{array}$ \\
\hline $\begin{array}{l}\text { Vacinação contra hepatite B e Anti- } \\
\text { HBS entre trabalhadores da saúde. }\end{array}$ & $\begin{array}{c}\text { Bahia (Santo Antônio de } \\
\text { Jesus). }\end{array}$ & $\begin{array}{l}\text { SOUZA FO, et al., } \\
2015 .\end{array}$ & Cad Saúde Colet. & $\begin{array}{l}\text { Transversal } \\
\text { (prevalência). }\end{array}$ & $\begin{array}{c}\text { Prevalência de } 59,9 \% \text { da } \\
\text { vacinação de três doses para } \\
\text { VHB, entre profissionais da } \\
\text { saúde. }\end{array}$ \\
\hline
\end{tabular}

Legenda: VHB: vírus da hepatite B. VHD: vírus da hepatite delta.

Fonte: Pimenta RSM, et al., 2020. 


\section{DISCUSSÃO}

Diante da análise dos estudos dessa revisão, pode-se constatar que a epidemiologia da hepatite B é bastante heterogênea no cenário nacional; áreas com dificuldade de acesso aos serviços de saúde, como as populações remanescentes de quilombos e indígenas alocados em áreas silvestres ou pouco urbanizadas, são desproporcionalmente afetadas. Assim, a Amazônia possui um caráter de alta endemicidade, explicada pela alta ocorrência da doença e suas consequências (BRASIL, 2017). De acordo com boletim epidemiológico divulgado em 2018, as cidades com maiores índices da doença são Porto Velho e Rio Branco; e o estado do Pará possui índices que ultrapassam o da capital, ambos situados na Amazônia (BRASIL, 2019).

A chamada Amazônia Legal, formada por 9 estados e 772 municípios, tem 27,5 milhões de habitantes, sendo que 400 mil são indígenas e há 170 etnias distintas. Consiste em uma população com oferta insuficiente de serviços públicos e com dificuldades em relação à infraestrutura, bem como comunicação e transportes (GARNELO L, 2019).

Nos indicadores sanitários, sociais e econômicos, há grande desigualdade quando comparada ao crescimento demográfico e econômico acelerado, com uma renda e condições de vida desfavoráveis para a maioria da população. Atualmente, quem vive com uma baixa renda, baixa escolaridade e sem acesso ao mercado de trabalho, depende do Sistema Único de Saúde (SUS). Além disso, a Política Nacional de Atenção Básica (PNAB - 2011 e 2017) preconiza uma implantação da Unidade Básica de Saúde Fluvial (UBSF), em que devem ser alocadas Equipes de Saúde da Família Ribeirinha, população tão carente de atenção; porém ainda enfrenta dificuldades com a retração de verbas públicas para a saúde (GARNELO L, 2019).

Diante do contexto epidemiológico, pôde-se observar uma estreita relação entre o Índice de Desenvolvimento Humano Municipal (IDHM) e a incidência de doenças infecciosas, como a hepatite B, sobretudo na região Norte, onde observa-se uma debilidade na condição e qualidade de vida dessa população (SOUZA FO, 2019).

Com o intuito de monitorar a doença e antever possíveis surtos, as hepatites virais estão inclusas na lista de doenças de notificação compulsória, ou seja, devem ser notificadas ao Sistema de Informação de Agravos de Notificação (SINAN), por um profissional de saúde. A partir desse registro é possível mapear e quantificar os casos no Brasil (FARIAS CS, 2019). Atualmente existem critérios para definição de pacientes contaminados, que são divulgados pelo Guia de Vigilância em Saúde e, no caso da hepatite B, podemos definir itens como, indivíduo que apresente um ou mais marcadores reagentes ou exame de biologia molecular para hepatite B: HBsAg reagente, Anti-HBc IgM reagente, HBV-DNA detectável (BRASIL, 2017).

$O$ vírus da hepatite $B$ determina uma variedade de apresentações clínicas, de portador assintomático a manifestações graves como hepatite fulminante, hepatite crônica, cirrose e carcinoma hepatocelular (CHC), (MARTINS M, 2018; IMMUNIZATION, 2018; KASPER, 2017; OLIVEIRA MS, 2019). Deve-se a isso, o fato de a hepatite $B$ ser uma doença silenciosa e comumente diagnosticada em estágios avançados, quando já há comprometimento de funções hepáticas (BRASIL, 2019).

A imunização contra o VHB foi um fator fundamental para reduzir os índices de prevalência da hepatite B no mundo. Esse modo de prevenção foi instituído na década de 90, inicialmente restrita a grupos de risco, perpassando para profissionais de saúde, e, enfim alcançando a população em geral, quando passou a ser oferecido gratuitamente pelo Sistema Único de Saúde (SUS) (INTERFARMA, 2016; GUSMÃO M, 2017). No Brasil, é preconizado que a vacinação seja feita em três doses no esquema de 0,30 e 180 dias (MARTINS AMEBL, 2015). Esta vacina tem demonstrado ser altamente imunogênica e segura (MARTINS M, 2018; IMMUNIZATION, 2018).

Entretanto, a implementação do programa vacinal não obteve o impacto esperado na região amazônica brasileira quando comparado a outros lugares, devido à baixa adesão da população (MARTINS AMEBL, 2015). Devido a isso, as estratégias de vacinação devem se concentrar em grupos de riscos específicos como bebês nascidos de mães e adolescentes HBsAg reagentes com o intuito de diminuir as taxas de prevalência de infecção e a transmissão vertical e horizontal do HBV. E após completar o esquema vacinal é válido verificar a soroconversão do indivíduo, ou seja, confirmar o desenvolvimento de anticorpos anti-HBS suficientes contra o vírus da hepatite B (GUSMÃO M, 2017). 
A partir da suspeita de infecção pelo HBV, o aparecimento de marcadores sorológicos do vírus será essencial no estabelecimento do diagnóstico da doença, uma vez que este depende basicamente do HBsAg e do anti-HBc IgM (BRASIL, 2015). Porém, vale ressaltar também que a hepatite $B$ pode ser classificada como oculta, quando da presença do VHB-DNA no fígado e presença ou ausência de detecção no soro com HBsAg(NUNES HM, 2017).

Em relação ao diagnóstico sorológico, quando o HBsAg, Anti-HBc total e Anti-HBs tem como resultado não reagente interpreta-se que o indivíduo não teve contato prévio com o HBV e por isso está susceptível a infecção pelo mesmo. Entretanto, quando o $\mathrm{HBsAg}$ tem como resultado não reagente e o Anti-HBc total e Anti-HBs reagente significa que houve imunização após infecção pelo HBV. Já o HBsAg e o Anti-HBc total não reagentes e o Anti-HBs reagente traduz-se que há imunização após vacinação contra o HBV. E por fim, para considerar o indivíduo infectado, os testes sorológicos devem possuir os seguintes resultados: HBsAG e Anti-HBc total reagentes e Anti-HBs não reagente (BRASIL, 2015).

A respeito do tratamento específico para a hepatite aguda, caracterizada por episódios de náuseas, vômitos e prurido, recomenda-se repouso até a normalização das aminotransferases, transaminase glutâmica oxalacética (TGO) e transaminase glutâmica pirúvica (TGP), dieta balanceada (pobre em gordura), suspensão da ingestão de bebidas alcoólicas por, no mínimo, seis meses, além da suspensão de medicamentos que estejam sendo usados sem orientação médica para que não haja agravo do dano hepático (GOIÁS, 2015).

Sobre o tratamento da hepatite crônica, o principal objetivo é reduzir o risco de progressão da doença hepática e seus desfechos, especificamente a cirrose, o carcinoma hepatocelular (CHC) e, consequentemente, a morte. Para estes pacientes, torna-se necessária a realização de biópsia hepática para avaliar a indicação de tratamento específico (CRUZ AS, 2018).

De acordo com American Association for the Study of Liver Diseases (AASLD), as indicações para o tratamento da hepatite B crônica são: HBsAg positivo por mais de seis meses; HBeAg positivo ou HBV-DNA acima de 30 mil cópias/ml (fase de replicação); TGP duas vezes acima do limite superior da normalidade, isto é, acima de $56 \mathrm{U} / \mathrm{L}$. Além disso, biópsia hepática com atividade inflamatória moderada a intensa (> A2) e/ou fibrose moderada a intensa (> F1), segundo critério da Sociedade Brasileira de Patologia/Metavir (BRASIL, 2017; TERRAULT NA, 2018).

Em relação ao esquema terapêutico, de acordo com o Protocolo Clínico e Diretrizes Terapêuticas, do Ministério da Saúde, de 2017, introduz-se ao SUS a alfapeguinterferona e engloba ainda mais os medicamentos entecavir e tenofovir. As novas opções medicamentosas oferecem maior facilidade posológica e menos efeitos adversos. Os medicamentos são: Alfapeguinterferona 2 a $40 \mathrm{KDa} 180 \mathrm{mcg} / \mathrm{semana}$ via subcutânea (SC); Alfapeguinterferona 2b $12 \mathrm{KDa} 1,5 \mathrm{mcg} / \mathrm{kg} / \mathrm{semana}$ via SC; Entecavir 0,5 mg 0,5-1,0 mg/dia via oral (VO), mais indicado para pacientes contraindicados a tenofovir ou com alterações renais; e Tenofovir (fumarato de tenofovir desoproxila) $300 \mathrm{mg}-300 \mathrm{mg} /$ dia VO, considerado a primeira linha de tratamento (BRASIL, 2017; SIEDERDISSEN CHZ, 2016). Os medicamentos citados funcionam como moduladores da resposta imunológica, com efeitos antiviral, antiproliferativo e imunomodulador (NAKANO LA, 2018; SILVA ALS, 2018; SIEDERDISSEN CHZ, 2016).

De acordo com a atualização da American Association for the Study of Liver Diseases (AASLD), relatada anteriormento, inclui o tenefovir alafenamida (TAF) à lista de terapias preferidas contra o vírus da hepatite $\mathrm{B}$, juntamente com entecavir, tenofovir disoproxil fumarato (TDF) e peginterferon (peg - IFN). Além disso, estudos sobre o uso de TDF para prevenção da transmissão de mãe para filho o levaram a ser elevado ao nível de terapia preferencial nesse cenário (TERRAULT NA, 2018).

Entende-se que a grande diferença no desfecho dos tratamentos e na evolução da hepatite crônica esteja relacionada às características virais. Diante disso, alguns genótipos apresentam melhor resposta terapêutica com o uso do interferon-alfa; outros já não respondem de forma tão positiva, possuindo assim um maior potencial carcinogênico. Porém, até então, faltam evidências que suportem a escolha da terapêutica em relação à genotipagem do HBV (BRASIL, 2017). 
O benefício dos tratamentos aplicados é verificado pela mudança no perfil sorológico, nas aminotransferases (TGO/TGP) e pelos níveis de HBV-DNA dos pacientes. Indivíduos portadores de hepatite B crônica HBeAg reagente, devem realizar os exames HBsAg, anti-HBs, HBeAg, anti-HBe e HBV-DNA anualmente; enquanto aqueles com HBeAg não reagente, devem realizar apenas os exames $\mathrm{HBsAg}$, antiHBs, HBV-DNA, também anualmente. Por fim, pacientes em tratamento com alfapeguinterferona devem fazer monitoramento clínico, incluindo hemograma completo a cada 12 semanas; AST/TGO e ALT/TGP na $2^{\text {a }}$ semana de tratamento e a cada quatro semanas de tratamento; glicemia de jejum, TSH (hormônio estimulante da tireoide) e T4 livre (tiroxina circulante no sangue) a cada 12 semanas (BRASIL, 2017).

Mesmo com a realização do tratamento, alguns indivíduos podem vir a reativar o vírus da hepatite $B$, isso pode ocorrer quando os mesmos são submetidos à quimioterapia ou estão fazendo uso de imunossupressores para transplantes ou em uso de esteroides em alta, média ou baixa dose associados ou não a outros imunossupressores. Devido a isso são observados o HBsAg e o anti-HbC para realização da estratificação de risco de reativação do VHB; se alto risco, realizar profilaxia; médio risco, realizar profilaxia ou observação e; se baixo risco, apenas observação (BRASIL, 2017).

É válido ressaltar que a interrupção do tratamento deve ser feita independente do esquema terapêutico utilizado, em caso de: eventos adversos importantes; ausência de adesão ao tratamento; alguma situação que contraindique formalmente a modalidade de tratamento, como por exemplo gestação; ou alguma situação que demande substituição do tratamento a exemplo de paciente com insuficiência hepática (BRASIL, 2017).

Recentemente, um estudo apresentado na AASLD Liver Meeting em Boston mostrou a eficácia de uma vacina terapêutica experimental (NASVAC). Essa vacina é administrada por via nasal para tratar pacientes infectados, e tem como alvo dois antígenos diferentes do vírus da hepatite $B$ (HBV) sendo um de superfície (HBsAg) e o outro central ( $\mathrm{HBcAg}$ ) versus interferon peguilado (Peg-IFN) em pacientes com hepatite B crônica. Pode-se concluir que o NASVAC induziu redução superior da carga viral quando comparado ao tratamento com Peg-IFN, mostrando ser uma alternativa eficaz de tratamento antiviral para pacientes com hepatite B crônica (MAHTAB MA, 2018).

A infecção por hepatite B pode levar a complicações graves em longo prazo, incluindo carcinoma hepatocelular e insuficiência hepática, especialmente quando adquirida na infância. No caso da gestante portadora de infecção crônica por HBV e perfil sorológico positivo, que não tenha realizado a imunoprofilaxia adequada no momento do parto pode comprometer o recém-nascido desenvolvendo infecção aguda por HBV, com progressão para infecção crônica ou também, complicações da doença hepática crônica na idade adulta. (BRASIL, 2019). De fato, atualmente, a vacinação é a única estratégia eficaz disponível para prevenir tal infecção (MARTINS M, 2018; IMMUNIZATION, 2018).

\section{CONSIDERAÇÕES FINAIS}

Diante do exposto, conclui-se que a hepatite B é um grave problema de saúde no Brasil, sobretudo, na região Amazônica, com altas taxas de incidência. Além disso, percebe-se que mesmo com a introdução de vacinas contra o vírus da hepatite $B$, nas últimas três décadas, às quais foram responsáveis por muitos avanços, ainda se observam altas taxas de ausência de imunização e vacinação incompleta, devido ao déficit na capacitação e a baixa escolaridade. Destaca-se, assim, a necessidade da avaliação dos programas de prevenção, assim como a implantação de políticas a fim de reduzir a hepatite B na região. Dessa forma, é essencial investimentos na prevenção e promoção de saúde, visando identificar falhas e aprimorar medidas necessárias para o controle da doença.

\section{REFERÊNCIAS}

1. BRASIL. Ministério da Saúde. Boletim epidemiológico - Sistema de vigilância em Saúde; 2019.

2. BRASIL. Ministério da Saúde. Manual técnico para o diagnóstico das hepatites virais. 2015.

3. BRASIL. Ministério da Saúde. Protocolo Clínico e Diretrizes Terapêuticas para Hepatite B e Coinfecções. 2017.

4. BRASIL. Ministério da Saúde. Protocolo Clínico e Diretrizes Terapêuticas para Prevenção da Transmissão Vertical de HIV, Sífilis e Hepatites Virais. Brasília-DF; 2019.

5. BRASIL. Sistema de Informação de Agravos de Notificação (SINAN). 2016. 
6. CRUZ AS, et al. Data survey between the 2010 one hepatitis 2016 b period in the municipality of Rolim de Moura. Brazilian Journal of Surgery and Clinical Research - BJSCR; 2018; 2317-4404.

7. FARIAS CS, et al. As Hepatites Virais no Brasil: uma análise a partir dos seus territórios. R. Ra'e Ga. Curitiba, v.46, p.90-109, Mar/2019; 10.5380.

8. GARNELO L. Especificidades e desafios das políticas públicas de saúde na Amazônia. Cad. Saúde Pública, Rio de Janeiro, v. 35, n. 12, e00220519, 2019.

9. GOIÁS. Secretaria de Estado da Saúde; Secretaria de Estado da Mulher, do Desenvolvimento Social, da Igualdade Racial, dos Direitos Humanos e do Trabalho. Programa Bolsa Família na Saúde: promoção da integralidade do cuidado aos titulares de direito do programa bolsa família / Secretaria de Estado da saúde de Goiás; Secretaria Cidadã; Goiânia: SUVISA/GVE/CVN, 2015.

10. GUSMÃO BM, et al. Análise do perfil sociodemográfico de notificados para hepatite B e imunização contra a doença. J. res.: fundam. care. online 2017; 9(3): 627-633.

11. IMMUNIZATION ACTION COALLITION, 2018. Vacuna contra la hepatites B: lo que necessita saber. Declaración informativa sobre vacunas. Spanish, 2018.

12. INTERFARMA. Associação da Indústria Farmacêutica de Pesquisa. Como as vacinas mudaram um país. São Paulo: Ativaonline Editora, 2016.

13. KASPER D, et al. Manual de Medicina de Harrison. 19aㅡ ed; vol 2; 2017.

14. MAHTAB MA, et al. Treatment of chronic hepatites $B$ naive patients with a therapeutic vaccine containing $H B s$ and HBc antigens (a randomized, open and treatment controlled phase III clinical trial). PLoS One. 2018;13(8):e0201236.

15. MARTINS AMEBL, et al. Fatores associados à imunização contra Hepatite B entre trabalhadores da Estratégia Saúde da Família. Rev Bras Enferm. 2015 jan-fev;68(1):84-92.

16. MARTINS M, et al. Positive hepatites $B$ surfasse antigen after vaccination in a newborn. Nascer e Crescer birth and growth medical journal year 2018, 27(2), 105-107.

17. NAKANO LA, et al. Assessment of the prevalence of vertical hepatitis $B$ transmission in two consecutive generations. Rev Assoc Med Bras 2018; 64(2):154-158.

18. NUNES HM, et al. Infecção oculta pelo vírus da hepatite $B$ em comunidade amazônica submetida a intenso fluxo migratório, estado do Pará, Brasil. Rev Pan-Amaz Saúde, 2017, 8(3):35-49.

19. OLIVEIRA MS, et al. Hepatitis B and Delta: clinical aspects of patients in the Brazilian Western Amazonia. Revista Brasileira de Enfermagem, 2019; 72(5):1265-70.

20. SANTANA CC, et al. Prevalence of occult hepatitis B in a population from the Brazilian Amazon region. J Med Virol, 2018; 90:1063-1070.

21. SIERDERDISSEN CHZ, CORNBERG M. Management of HBV and HBV/HDV-Associated liver cirrhosis. Visceral Medicine, 2016, Apr;32(2):86-94.

22. SILVA ALS, et al. Hepatites virais: B, C e D: atualização. Rev Bras Med. SP, 2013; 10(3):206-18.

23. SILVA RSU, et al. Prevalência da infecção pelo vírus da hepatite $B$ em um município do interior do estado do Acre, Amazônia Ocidental, Brasil. Rev Pan-Amaz Saúde, 2017; 8(3):19-26.

24. SOUTO FJD. Distribution of hepatitis B infection in Brazil: the epidemiological situation at the beginning of the $21 \mathrm{st}$ century. Revista da Sociedade Brasileira de Medicina Tropical; 2016; 49(1):11-23.

25. SOUZA FO, et al. Vacinação contra hepatite B e Anti-HBS entre trabalhadoroes da saúde. Cad. Saúde Colet., 2015, 23 (2): 172-179; 10.1590.

26. TERRAULT NA, et al. Update on Prevention, Diagnosis, and Treatment of Chronic Hepatitis B: AASLD 2018 Hepatitis B Guidance. AASLD Practice Guidelines HEPATOLOGY Honor Roll: Editor's Choice, vol. 67, NO. 4, 2018. 05, Feb. 2018. 